Annals of Pure and Applied Mathematics

Vol. 15, No. 1, 2017, 51-65

ISSN: 2279-087X (P), 2279-0888(online)

Published on 11 December 2017

www.researchmathsci.org

DOI: http://dx.doi.org/10.22457/apam.v15n1a5

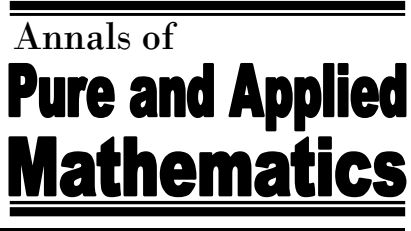

\title{
Wiener Vector, Hyper-Wiener Vector, Wiener Number and Hyper-Wiener Number of Molecular Graphs
}

\author{
P.Gayathri ${ }^{1}$ and T.Ragavan ${ }^{2}$ \\ ${ }^{1,2} \mathrm{PG}$ and Research Department of Mathematics \\ A.V.C.College (Autonomous), Mannampandal, Mayiladuthurai, Tamilnadu, India \\ pgayathrisundar@gmail.com and ${ }^{2}$ tragavanmaths@gmail.com
}

Received 3 November 2017; accepted 6 December 2017

Abstract. In this paper we have considered the popular topological indices Wiener vector, Hyper-Wiener Vector, Wiener Matrix Sequence, Hyper Wiener Matrix Sequence, Wiener Polynomial Sequence and Hyper-Wiener Polynomial, Wiener number and Hyper Wiener number of Benzenoid hydrocarbons molecular graphs are computed and presented in a well defined form. The correlation coefficient shows that the present findings are of highest accuracy to predict Log P.

Keywords: Molecular graph, Wiener matrix sequence, hyper-Wiener vector, weighted Wiener polynomial; Wiener polynomial sequence.

AMS Mathematics Subject Classification (2010): 92E10, 05C07, 05C10, $05 \mathrm{C} 12$

\section{Introduction}

Quantitative structure-Activity and Structure-property relationships (QSAR/QSPR) use chemo-metric methods to study how a given biological activity or a physiochemical property varies as a function of topological descriptors describing the chemical structure of the molecules. With these studies it is possible to replace costly and time taking biological tests or experiments of a given physiochemical property with models involving topological descriptors. In previous literature [1] designed a model to predict Lipophilicity of polyacenes with topological indices PI and Sd indices. In this paper we have considered the popular topological indices Wiener vector, Hyper-Wiener Vector, Wiener Matrix Sequence, Hyper Wiener Matrix Sequence, Wiener Polynomial Sequence and Hyper-Wiener Polynomial, Wiener number and Hyper Wiener number of Benzenoid hydrocarbons molecular graphs are computed. The correlation coefficient between the Wiener number and the corresponding $\log \mathrm{P}$ values are computed as well as the correlation coefficient between Hyper Wiener number and the corresponding $\log \mathrm{P}$ values are showed diagrammatically.

\section{Preliminaries}

In this section we list definitions that are required for the paper [2].

\subsection{Wiener vector of a graph $G$}

For a connected graph $\mathrm{G}$ with $\mathrm{n}$ vertices, denoted by $1,2 \ldots \mathrm{n}$, let 


\section{P.Gayathri and T.Ragavan}

$W_{k}=\sum_{i<j, d i j=k} d_{i j}, k=1,2, \ldots$ The vector $\left(\mathrm{W}_{1}, \mathrm{~W}_{2}, \ldots\right)$ is called the Wiener Vector of $\mathrm{G}$, denoted by WV (G).Clearly; the sum of all components of the Wiener vector of $G$ is just equal to the Wiener number of $\mathrm{G}$.

\subsection{Hyper Wiener vector of a graph $G$}

For a connected graph $\mathrm{G}$ with $\mathrm{n}$ vertices, denoted by $1,2 \ldots \mathrm{n}$, let $W_{i j, k}=\max \left\{d_{i j}-k+1,0\right\}$ where $\mathrm{d}_{\mathrm{ij}}$ is the distance between vertices $\mathrm{i}$ and $\mathrm{j}$. Then ${ }^{k} W=\sum_{i<j} W_{i j, k}, k=1,2, \ldots \ldots$ are called the hyper Wiener numbers of $\mathrm{G}$. The vector $\left({ }^{1} W,{ }^{2} W \ldots . ..\right)$ is called the hyper-Wiener vector of $\mathrm{G}$, denoted by HWV (G).

\subsection{A Wiener matrix Sequence}

Let $W^{(1)}=D$ for $\mathrm{k}=1,2 \ldots W^{(k+1)}$ is obtained from $W^{(k)}$ by leaving zeroes in place and replacing each nonzero entry $\mathrm{x}$ of $W^{(k)}$ by $\mathrm{x}-1$. Let $\mathrm{D}$ be the distance matrix of a connected graph $\mathrm{G}$, and let $W^{(1)}, W^{(2)}, \ldots \ldots .$. be the Wiener matrix sequence of $\mathrm{G}$.

\subsection{The hyper-Wiener matrix $W^{(H)}$ of $\mathbf{G}$}

$W^{(H)}=\sum_{k=1,2, \ldots} W^{(k)}$. From the above definitions, we can see that $(\mathrm{i}, \mathrm{j})$-entry of $W^{(k)}$ is just equal to $d_{i j}-k+1$, so the sum of entries of upper triangle of $W^{(k)}$ is just equal to ${ }^{k} W$.

Moreover, the sum of entries of upper triangle of the hyper-Wiener matrix $W^{(H)}$ is just equal to the hyper-Wiener number R. However, the hyper-Wiener matrix is applicable for any connected structure.

\subsection{Wiener polynomial of $\mathbf{G}$}

A generating function $W(G, x)$ related to the Wiener index of a graph $\mathrm{G}$, called the Wiener polynomial denoted by $W(G, x)=\sum_{\{u, v \subseteq V(G)\}} x^{d(u, v)}$, where $d(u, v)$ denotes the distance between vertices $u$ and $v$.

Let $\mathrm{D}$ be the distance matrix of a graph G. Let 1 be the largest entry of $D$, and let $2 \mathrm{~d}_{\mathrm{k}}$ be the number of such entries of $\mathrm{D}$ that are equal to $\mathrm{k}$.

\subsection{Wiener polynomial sequence of $\mathbf{G}$}

In a hyper-Wiener vector, ${ }^{I} W=W$ so we can call ${ }^{I} W=W$ the first Wiener number and ${ }^{k} W$ the $\mathrm{k}^{\text {th }}$ Wiener number.

According to the Wiener polynomial, we will introduce a Wiener polynomial sequence $W_{1}(G, x), W_{2}(G, x) \ldots$... of a graph $\mathrm{G}$, where the $\mathrm{k}^{\text {th }}$ Wiener polynomial of $\mathrm{G}$ is closely related to the $\mathrm{k}^{\text {th }}$ Wiener number. 


\section{Wiener Vector, Hyper-Wiener Vector, Wiener Number and Hyper-Wiener Number of Molecular Graphs}

Let $\mathrm{G}$ be a connected graph with $\mathrm{n}$ vertices. The $\mathrm{k}^{\text {th }}$ Wiener polynomial of $\mathrm{G}$, $1 \leq k \leq \operatorname{dia}(G) 1$, is defined by $W_{k}(G, x)=\sum_{\{u, v\} \subseteq V(G)} x^{\max [d(u, v)-k+1,0]}$, where dia $(\mathrm{G})$ is the diameter of $G$. The polynomial sequence $W_{1}(G, x), W_{2}(G, x), W_{3}(G, x) \ldots$ is called the Wiener polynomial sequence of $\mathrm{G}$.

$W_{1}(G, x)=W(G, x)$ and $W_{k}{ }^{\prime}(G, 1)={ }^{k} W$. The constant term of $\mathrm{W}_{\mathrm{k}}(\mathrm{G}, \mathrm{x})$ is equal to the number of the pairs of vertices with distances less than $\mathrm{k}$. For the hyper-Wiener vector of a graph $\mathrm{G}$, its components ${ }^{\mathrm{i}} \mathrm{W}$ and ${ }^{\mathrm{j}} \mathrm{W}$ might have different contributions in some research for the structure-property relationship.

\subsection{Weighted hyper-Wiener number}

$R_{W}(G)$ of a graph $\mathrm{G}$ is defined a $R_{W}(G)=\sum_{k=1,2, \ldots \ldots}{ }^{k} W y_{k}$, Where $y_{k}$ is the weight of ${ }^{k} W$.In addition; we will introduce a novel weighted hyper-Wiener polynomial $H W(G, x, y)$ of a graph $\mathrm{G}$, so that the hyper-Wiener vector and the weighted hyper-Wiener number can be obtained from the polynomial.

\subsection{Weighted hyper-Wiener polynomial}

$H W(G, x, y)$ of a graph $\mathrm{G}$ is defined as $H W(G, x, y)=\sum_{k=1,2, \ldots} W_{k}(G, x) . y_{k}$, where $\mathrm{y}=$ $\left(\mathrm{y}_{1}, \mathrm{y}_{2} \ldots\right) . \quad$ We have $\frac{d}{d x}[H W(G, x, y)] / x=1=R_{w}(G)=\sum_{k=1,2, \ldots}{ }^{k} W y_{k}$ where the coefficients of $y_{k}$ in the weighted hyper Wiener number $R_{w}(G)$ are equal to the $\mathrm{k}^{\text {th }}$ Wiener number. Hence, the hyper-Wiener vector can be given by coefficients of $\frac{d}{d x}[H W(G, x, y)] / x=1$.

\subsection{Hyper Wiener number}

In particular, we have $\frac{d}{d x}[H W(G, x, y)] / x=1, y=(1,1,1 \ldots \ldots)=R(G)$

Lipophilicity or Hydrophobicity: [3]

The partition coefficient, abbreviated $\boldsymbol{P}$, is defined as a particular ratio of the concentrations of a solute between the two solvents (a biphase of liquid phases), specifically for un-ionized solutes, and the logarithm of the ratio is thus $\log \boldsymbol{P}$. When one of the solvents is water and the other is a non-polar solvent, then the $\log P$ value is a measure of lipophilicity or hydrophobicity.

\section{Computational methodology}

The Benzenoid molecular graph in the figure1 is composed of hexagonal rings that are face bounded by six-membered cycles in the plane. Any two rings have either one common edge or have no common vertices. 


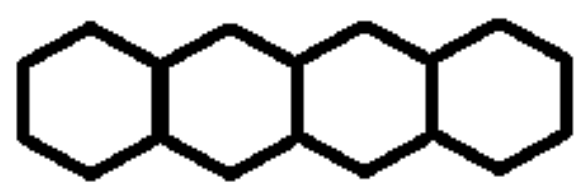

Figure 1: The benzenoid hydrocarbon molecular graph

For graph $\mathrm{G}$ in Figure 2, its Wiener matrix sequence, Wiener vector, and hyper-Wiener vector, Wiener polynomial sequence and the weighted hyper Wiener polynomial of $\mathrm{G}$ are determined and given below:

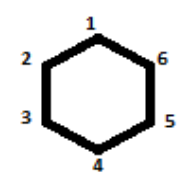

Figure 2: Benzene

$\mathrm{W}^{(1)}(\mathrm{G})=\left(\begin{array}{cccccc}0 & 1 & 2 & 3 & 2 & 1 \\ 1 & 0 & 1 & 2 & 3 & 2 \\ 2 & 1 & 0 & 1 & 2 & 3 \\ 3 & 2 & 1 & 0 & 1 & 2 \\ 2 & 3 & 2 & 1 & 0 & 1 \\ 1 & 2 & 3 & 2 & 1 & 0\end{array}\right): \mathrm{W}^{(2)}(\mathrm{G})=\left(\begin{array}{cccccc}0 & 0 & 1 & 2 & 1 & 0 \\ 0 & 0 & 0 & 1 & 2 & 1 \\ 1 & 0 & 0 & 0 & 1 & 2 \\ 2 & 1 & 0 & 0 & 0 & 1 \\ 1 & 2 & 1 & 0 & 0 & 0 \\ 0 & 1 & 2 & 1 & 0 & 0\end{array}\right)$

$\mathrm{W}^{(3)}(\mathrm{G})=\left(\begin{array}{cccccc}0 & 0 & 0 & 1 & 0 & 0 \\ 0 & 0 & 0 & 0 & 1 & 0 \\ 0 & 0 & 0 & 0 & 1 & 0 \\ 1 & 0 & 0 & 0 & 0 & 0 \\ 0 & 1 & 0 & 0 & 0 & 0 \\ 0 & 0 & 1 & 0 & 0 & 0\end{array}\right) ; \mathrm{W}^{(\mathrm{H})}(\mathrm{G})=\left(\begin{array}{cccccc}0 & 1 & 3 & 6 & 3 & 1 \\ 1 & 0 & 1 & 3 & 6 & 3 \\ 3 & 1 & 0 & 1 & 3 & 6 \\ 6 & 3 & 1 & 0 & 1 & 3 \\ 3 & 6 & 3 & 1 & 0 & 1 \\ 1 & 3 & 6 & 3 & 1 & 0\end{array}\right)$

$\mathrm{WV}(\mathrm{G})=(6,12,9), \operatorname{HWV}(\mathrm{G})=(27,12,3), \mathrm{W}=27, \mathrm{R}=42$

$W^{\prime}(G, x)=9 x^{2}+12 x+6$

$W^{(1)}(G, x)=3 x^{3}+6 x^{2}+6 x$.

$W^{(2)}(G, x)=3 x^{2}+6 x+6$

$W^{(3)}(G, x)=3 x+12$

$H W(G, x, y)=\left(3 x^{3}+6 x^{2}+6 x\right) y_{1}+\left(3 x^{2}+6 x+6\right) y_{2}+(3 x+12) y_{3}$

$\frac{d}{d x} H W(G, x, y) / x=1=27 y_{1}+12 y_{2}+3 y_{3}$ 
Wiener Vector, Hyper-Wiener Vector, Wiener Number and Hyper-Wiener Number of Molecular Graphs

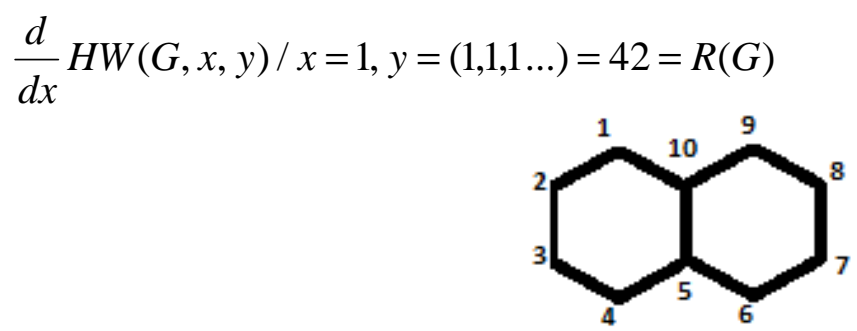

Figure 3: Napthalene

For graph G in Figure 3, its Wiener matrix sequence, Wiener vector, and hyper-Wiener vector, Wiener polynomial sequence and the weighted hyper Wiener polynomial of $\mathrm{G}$ are determined and given below:

$$
\mathrm{W}^{(1)}(\mathrm{G})=\left(\begin{array}{cccccccccc}
0 & 1 & 2 & 3 & 2 & 3 & 4 & 3 & 2 & 1 \\
1 & 0 & 1 & 2 & 3 & 4 & 5 & 4 & 3 & 2 \\
2 & 1 & 0 & 1 & 2 & 3 & 4 & 5 & 4 & 3 \\
3 & 2 & 1 & 0 & 1 & 2 & 3 & 4 & 3 & 2 \\
2 & 3 & 2 & 1 & 0 & 1 & 2 & 3 & 2 & 1 \\
3 & 4 & 3 & 2 & 1 & 0 & 1 & 2 & 3 & 2 \\
4 & 5 & 4 & 3 & 2 & 1 & 0 & 1 & 2 & 3 \\
3 & 4 & 5 & 4 & 3 & 2 & 1 & 0 & 1 & 2 \\
2 & 3 & 4 & 3 & 2 & 3 & 2 & 1 & 0 & 1 \\
1 & 2 & 3 & 2 & 1 & 2 & 3 & 2 & 1 & 0
\end{array}\right)
$$


P.Gayathri and T.Ragavan

$$
\mathrm{W}^{(3)}(\mathrm{G})=\left(\begin{array}{cccccccccc}
0 & 0 & 0 & 1 & 0 & 1 & 2 & 1 & 0 & 0 \\
0 & 0 & 0 & 0 & 1 & 2 & 3 & 2 & 1 & 0 \\
0 & 0 & 0 & 0 & 0 & 1 & 2 & 3 & 2 & 1 \\
1 & 0 & 0 & 0 & 0 & 0 & 1 & 2 & 1 & 0 \\
0 & 1 & 0 & 0 & 0 & 0 & 0 & 1 & 0 & 0 \\
1 & 2 & 1 & 0 & 0 & 0 & 0 & 0 & 1 & 0 \\
2 & 3 & 2 & 1 & 0 & 0 & 0 & 0 & 0 & 1 \\
1 & 2 & 3 & 2 & 1 & 0 & 0 & 0 & 0 & 0 \\
0 & 1 & 2 & 1 & 0 & 1 & 0 & 0 & 0 & 0 \\
0 & 0 & 1 & 0 & 0 & 0 & 1 & 0 & 0 & 0
\end{array}\right)
$$


Wiener Vector, Hyper-Wiener Vector, Wiener Number and Hyper-Wiener Number of Molecular Graphs

$$
\mathrm{W}^{(\mathrm{H})}(\mathrm{G})=\left(\begin{array}{cccccccccc}
0 & 1 & 3 & 6 & 3 & 6 & 10 & 6 & 3 & 1 \\
1 & 0 & 1 & 3 & 6 & 10 & 15 & 10 & 6 & 3 \\
3 & 1 & 0 & 1 & 3 & 6 & 10 & 15 & 10 & 6 \\
6 & 3 & 1 & 0 & 1 & 3 & 6 & 10 & 6 & 3 \\
3 & 6 & 3 & 1 & 0 & 1 & 3 & 6 & 3 & 1 \\
6 & 10 & 6 & 3 & 1 & 0 & 1 & 3 & 6 & 3 \\
10 & 15 & 10 & 6 & 3 & 1 & 0 & 1 & 3 & 6 \\
6 & 10 & 15 & 10 & 6 & 3 & 1 & 0 & 1 & 3 \\
3 & 6 & 10 & 6 & 3 & 6 & 3 & 1 & 0 & 1 \\
1 & 3 & 6 & 3 & 1 & 3 & 6 & 3 & 1 & 0
\end{array}\right)
$$

$\mathrm{WV}(\mathrm{G})=(11,28,36,24,10) ; \operatorname{HWV}(\mathrm{G})=(109,64,30,10,2)$

$\mathrm{W}=109, \mathrm{R}=215$.

$$
\begin{aligned}
& W^{\prime}(G, x)=10 x^{4}+24 x^{3}+36 x^{2}+28 x+11 \\
& W^{(1)}(G, x)=2 x^{5}+6 x^{4}+12 x^{3}+14 x^{2}+11 x \\
& W^{(2)}(G, x)=2 x^{4}+6 x^{3}+12 x^{2}+14 x+11 \\
& W^{(3)}(G, x)=2 x^{3}+6 x^{2}+12 x+25 \\
& W^{(4)}(G, x)=2 x^{2}+6 x+37 \\
& W^{(5)}(G, x)=2 x+43 \\
& H W(G, x, y)=\left(2 x^{5}+6 x^{4}+12 x^{3}+14 x^{2}+11 x\right) y_{1}+\left(2 x^{4}+6 x^{3}+12 x^{2}+14 x+11\right) y_{2}+ \\
& \left.\begin{array}{r}
\left(2 x^{3}+6 x^{2}+12 x+25\right) y_{3}+\left(2 x^{2}+6 x+37\right) y_{4}+(2 x+43) y_{5} \\
\frac{d}{d x} H W(G, x, y) / x=1=\left[\begin{array}{l}
\left(10 x^{4}+24 x^{3}+36 x^{2}+28 x+11\right) y_{1}+ \\
\left(8 x^{3}+18 x^{2}+24 x+14\right) y_{2} \\
+\left(6 x^{2}+12 x+12\right) y_{3}+ \\
(4 x+6) y_{4}+2 y_{5}
\end{array}\right] \\
=109 y_{1}+64 y_{2}+30 y_{3}+10 y_{4}+2 y_{5}
\end{array}\right] \\
& \frac{d}{d x} H W(G, x, y) / x=1, y=(1,1,1,1,1 \ldots)=215=R(G)
\end{aligned}
$$


P.Gayathri and T.Ragavan

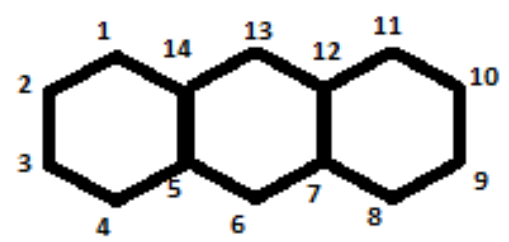

Figure 4: Anthracene

For graph G in Figure 4, its Wiener matrix sequence, Wiener vector, and hyper-Wiener vector, Wiener polynomial sequence and the weighted hyper Wiener polynomial of $\mathrm{G}$ are determined and given below:

$$
\mathrm{W}^{(1)}(\mathrm{G})=\left(\begin{array}{cccccccccccccc}
0 & 1 & 2 & 3 & 2 & 3 & 4 & 5 & 6 & 5 & 4 & 3 & 2 & 1 \\
- & 0 & 1 & 2 & 3 & 4 & 5 & 6 & 7 & 6 & 5 & 4 & 3 & 2 \\
- & - & 0 & 1 & 2 & 3 & 4 & 5 & 6 & 7 & 6 & 5 & 4 & 3 \\
- & - & - & 0 & 1 & 2 & 3 & 4 & 5 & 6 & 5 & 4 & 3 & 2 \\
- & - & - & - & 0 & 1 & 2 & 3 & 4 & 5 & 4 & 3 & 2 & 1 \\
- & - & - & - & - & 0 & 1 & 2 & 3 & 4 & 3 & 2 & 3 & 2 \\
- & - & - & - & - & - & 0 & 1 & 2 & 3 & 2 & 1 & 2 & 3 \\
- & - & - & - & - & - & - & 0 & 1 & 2 & 3 & 2 & 3 & 4 \\
- & - & - & - & - & - & - & - & 0 & 1 & 2 & 3 & 4 & 5 \\
- & - & - & - & - & - & - & - & - & 0 & 1 & 2 & 3 & 4 \\
- & - & - & - & - & - & - & - & - & - & 0 & 1 & 2 & 3 \\
- & - & - & - & - & - & - & - & - & - & - & 0 & 1 & 2 \\
- & - & - & - & - & - & - & - & - & - & - & - & 0 & 1 \\
- & - & - & - & - & - & - & - & - & - & - & - & & 0
\end{array}\right)
$$


Wiener Vector, Hyper-Wiener Vector, Wiener Number and Hyper-Wiener Number of Molecular Graphs

$$
\mathrm{W}^{(2)}(\mathrm{G})=\left(\begin{array}{cccccccccccccc}
0 & 0 & 1 & 2 & 1 & 2 & 3 & 4 & 5 & 4 & 3 & 2 & 1 & 0 \\
- & 0 & 0 & 1 & 2 & 3 & 4 & 5 & 6 & 5 & 4 & 3 & 2 & 1 \\
- & - & 0 & 0 & 1 & 2 & 3 & 4 & 5 & 6 & 5 & 4 & 3 & 2 \\
- & - & - & 0 & 0 & 1 & 2 & 3 & 4 & 5 & 4 & 3 & 2 & 1 \\
- & - & - & - & 0 & 0 & 1 & 2 & 3 & 4 & 3 & 2 & 1 & 0 \\
- & - & - & - & - & 0 & 0 & 1 & 2 & 3 & 2 & 1 & 2 & 1 \\
- & - & - & - & - & - & 0 & 0 & 1 & 2 & 1 & 0 & 1 & 2 \\
- & - & - & - & - & - & - & 0 & 0 & 1 & 2 & 1 & 2 & 3 \\
- & - & - & - & - & - & - & - & 0 & 0 & 1 & 2 & 3 & 4 \\
- & - & - & - & - & - & - & - & - & 0 & 0 & 1 & 2 & 3 \\
- & - & - & - & - & - & - & - & - & - & 0 & 0 & 1 & 2 \\
- & - & - & - & - & - & - & - & - & - & - & 0 & 0 & 1 \\
- & - & - & - & - & - & - & - & - & - & - & - & 0 & 0 \\
- & - & - & - & - & - & - & - & - & - & - & - & & 0
\end{array}\right)
$$


P.Gayathri and T.Ragavan

$$
\mathrm{W}^{(4)}(\mathrm{G})=\left(\begin{array}{cccccccccccccc}
0 & 0 & 0 & 0 & 0 & 0 & 1 & 2 & 3 & 2 & 1 & 0 & 0 & 0 \\
- & 0 & 0 & 0 & 0 & 1 & 2 & 3 & 4 & 3 & 2 & 1 & 0 & 0 \\
- & - & 0 & 0 & 0 & 0 & 1 & 2 & 3 & 4 & 3 & 2 & 1 & 0 \\
- & - & - & 0 & 0 & 0 & 0 & 1 & 2 & 3 & 2 & 1 & 0 & 0 \\
- & - & - & - & 0 & 0 & 0 & 0 & 1 & 2 & 1 & 0 & 0 & 0 \\
- & - & - & - & - & 0 & 0 & 0 & 0 & 1 & 0 & 0 & 0 & 0 \\
- & - & - & - & - & - & 0 & 0 & 0 & 0 & 0 & 0 & 0 & 0 \\
- & - & - & - & - & - & - & 0 & 0 & 0 & 0 & 0 & 0 & 1 \\
- & - & - & - & - & - & - & - & 0 & 0 & 0 & 0 & 1 & 2 \\
- & - & - & - & - & - & - & - & - & 0 & 0 & 0 & 0 & 1 \\
- & - & - & - & - & - & - & - & - & - & 0 & 0 & 0 & 0 \\
- & - & - & - & - & - & - & - & - & - & - & 0 & 0 & 0 \\
- & - & - & - & - & - & - & - & - & - & - & - & 0 & 0 \\
- & - & - & - & - & - & - & - & - & - & - & - & - & 0
\end{array}\right) .
$$

$$
\mathrm{W}^{(5)}(\mathrm{G})=\left(\begin{array}{cccccccccccccc}
0 & 0 & 0 & 0 & 0 & 0 & 0 & 1 & 2 & 1 & 0 & 0 & 0 & 0 \\
- & 0 & 0 & 0 & 0 & 0 & 1 & 2 & 3 & 2 & 1 & 0 & 0 & 0 \\
= & = & 0 & 0 & 0 & 0 & 0 & 1 & 2 & 3 & 2 & 1 & 0 & 0 \\
= & = & - & 0 & 0 & 0 & 0 & 0 & 1 & 2 & 1 & 0 & 0 & 0 \\
- & - & - & - & 0 & 0 & 0 & 0 & 0 & 1 & 0 & 0 & 0 & 0 \\
- & - & - & - & - & 0 & 0 & 0 & 0 & 0 & 0 & 0 & 0 & 0 \\
- & - & - & - & - & - & 0 & 0 & 0 & 0 & 0 & 0 & 0 & 0 \\
- & - & - & - & - & - & - & 0 & 0 & 0 & 0 & 0 & 0 & 0 \\
- & - & - & - & - & - & - & - & 0 & 0 & 0 & 0 & 0 & 1 \\
- & - & - & - & - & - & - & - & - & 0 & 0 & 0 & 0 & 0 \\
- & - & - & - & - & - & - & - & - & - & 0 & 0 & 0 & 0 \\
- & - & - & - & - & - & - & - & - & - & - & 0 & 0 & 0 \\
- & - & - & - & - & - & - & - & - & - & - & - & 0 & 0 \\
- & - & - & - & - & - & - & - & - & - & - & - & - & 0
\end{array}\right)
$$


Wiener Vector, Hyper-Wiener Vector, Wiener Number and Hyper-Wiener Number of Molecular Graphs

$$
\mathrm{W}^{(6)}(\mathrm{G})=\left(\begin{array}{cccccccccccccc}
0 & 0 & 0 & 0 & 0 & 0 & 0 & 0 & 1 & 0 & 0 & 0 & 0 & 0 \\
- & 0 & 0 & 0 & 0 & 0 & 0 & 1 & 2 & 1 & 0 & 0 & 0 & 0 \\
- & - & 0 & 0 & 0 & 0 & 0 & 0 & 1 & 2 & 1 & 0 & 0 & 0 \\
- & - & - & 0 & 0 & 0 & 0 & 0 & 0 & 1 & 0 & 0 & 0 & 0 \\
- & - & - & - & 0 & 0 & 0 & 0 & 0 & 0 & 0 & 0 & 0 & 0 \\
- & - & - & - & - & 0 & 0 & 0 & 0 & 0 & 0 & 0 & 0 & 0 \\
- & - & - & - & - & - & 0 & 0 & 0 & 0 & 0 & 0 & 0 & 0 \\
- & - & - & - & - & - & - & 0 & 0 & 0 & 0 & 0 & 0 & 0 \\
- & - & - & - & - & - & - & - & 0 & 0 & 0 & 0 & 0 & 0 \\
- & - & - & - & - & - & - & - & - & 0 & 0 & 0 & 0 & 0 \\
- & - & - & - & - & - & - & - & - & - & 0 & 0 & 0 & 0 \\
- & - & - & - & - & - & - & - & - & - & - & 0 & 0 & 0 \\
- & - & - & - & - & - & - & - & - & - & - & - & 0 & 0 \\
- & - & - & - & - & - & - & - & - & - & - & - & - & 0
\end{array}\right)
$$


P.Gayathri and T.Ragavan

$$
\mathrm{W}^{(\mathrm{H})}(\mathrm{G})=\left(\begin{array}{cccccccccccccc}
0 & 1 & 3 & 6 & 3 & 6 & 10 & 15 & 21 & 15 & 10 & 6 & 3 & 1 \\
- & 0 & 1 & 3 & 6 & 10 & 15 & 21 & 28 & 21 & 15 & 10 & 6 & 3 \\
- & - & 0 & 1 & 3 & 6 & 10 & 15 & 21 & 28 & 21 & 15 & 10 & 6 \\
- & - & - & 0 & 1 & 3 & 6 & 10 & 15 & 21 & 15 & 10 & 6 & 3 \\
- & - & - & - & 0 & 1 & 3 & 6 & 10 & 15 & 10 & 6 & 3 & 1 \\
- & - & - & - & - & 0 & 1 & 3 & 6 & 10 & 6 & 3 & 6 & 3 \\
- & - & - & - & - & - & 0 & 1 & 3 & 6 & 3 & 1 & 3 & 6 \\
- & - & - & - & - & - & - & 0 & 1 & 3 & 6 & 3 & 6 & 10 \\
- & - & - & - & - & - & - & - & 0 & 1 & 3 & 6 & 10 & 15 \\
- & - & - & - & - & - & - & - & - & 0 & 1 & 3 & 6 & 10 \\
- & - & - & - & - & - & - & - & - & - & 0 & 1 & 3 & 6 \\
- & - & - & - & - & - & - & - & - & - & - & 0 & 1 & 3 \\
- & - & - & - & - & - & - & - & - & - & - & - & 0 & 1 \\
- & - & - & - & - & - & - & - & - & - & - & - & - & 0
\end{array}\right)
$$

$$
\begin{aligned}
& \text { WV }(\mathrm{G})=(16,44,63,56,50,36,14) ; \mathrm{HWV}(\mathrm{G})=(279,188,113,60,28,10,2) \\
& \mathrm{W}=279, \mathrm{R}=680 \\
& W^{\prime}(G, x)=14 x^{6}+36 x^{5}+50 x^{4}+56 x^{3}+63 x^{2}+44 x+16 \\
& W^{(1)}(G, x)=2 x^{7}+6 x^{6}+10 x^{5}+14 x^{4}+21 x^{3}+22 x^{2}+16 x \\
& W^{(2)}(G, x)=2 x^{6}+6 x^{5}+10 x^{4}+14 x^{3}+21 x^{2}+22 x+16 \\
& W^{(3)}(G, x)=2 x^{5}+6 x^{4}+10 x^{3}+14 x^{2}+21 x+38 \\
& W^{(4)}(G, x)=2 x^{4}+6 x^{3}+10 x^{2}+14 x+59 \\
& W^{(5)}(G, x)=2 x^{3}+6 x^{2}+10 x+73 \\
& W^{(6)}(G, x)=2 x^{2}+6 x+83 \\
& W^{(7)}(G, x)=2 x+89 \\
& H W(G, x, y)=\left(2 x^{7}+6 x^{6}+10 x^{5}+14 x^{4}+21 x^{3}+22 x^{2}+16 x\right) y_{1}+ \\
& \left(2 x^{6}+6 x^{5}+10 x^{4}+14 x^{3}+21 x^{2}+22 x+16\right) y_{2}+\left(2 x^{5}+6 x^{4}+10 x^{3}+14 x^{2}+21 x+38\right) y_{3}+ \\
& \left(2 x^{4}+6 x^{3}+10 x^{2}+14 x+59\right) y_{4}+\left(2 x^{3}+6 x^{2}+10 x+73\right) y_{5}+\left(2 x^{2}+6 x+83\right) y_{6}+ \\
& (2 x+89) y_{7} \\
& \frac{d}{d x} H W(G, x, y) / x=1, y=(1,1,1,1,1 \ldots .)=680=R(G)
\end{aligned}
$$


Wiener Vector, Hyper-Wiener Vector, Wiener Number and Hyper-Wiener Number of Molecular Graphs

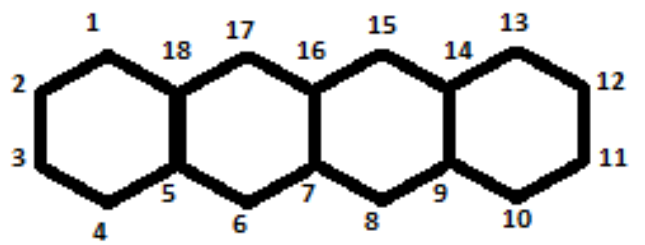

Figure 5: Tetracene

Similarly, for graph G in Figure 5 we can determine by Wiener vector and hyper-Wiener vector from Wiener matrix sequence and are given below:

$\mathrm{WV}(\mathrm{G})=(21,60,90,88,90,84,70,48,18)$;

$\operatorname{HWV}(\mathrm{G})=(569,416,284,182,110,60,28,10,2)$

$\mathrm{W}=569, \mathrm{R}=1661$

$W^{\prime}(G, x)=18 x^{8}+48 x^{7}+70 x^{6}+84 x^{5}+90 x^{4}+88 x^{3}+90 x^{2}+60 x+21$

$W^{(1)}(G, x)=2 x^{9}+6 x^{8}+10 x^{7}+14 x^{6}+18 x^{5}+22 x^{4}+30 x^{3}+30 x^{2}+21 x$

$W^{(2)}(G, x)=2 x^{8}+6 x^{7}+10 x^{6}+14 x^{5}+18 x^{4}+22 x^{3}+30 x^{2}+30 x+21$

$W^{(3)}(G, x)=2 x^{7}+6 x^{6}+10 x^{5}+14 x^{4}+18 x^{3}+22 x^{2}+30 x+51$

$W^{(4)}(G, x)=2 x^{6}+6 x^{5}+10 x^{4}+14 x^{3}+18 x^{2}+22 x+81$

$W^{(5)}(G, x)=2 x^{5}+6 x^{4}+10 x^{3}+14 x^{2}+18 x+103$

$W^{(6)}(G, x)=2 x^{4}+6 x^{3}+10 x^{2}+14 x+121$

$W^{(7)}(G, x)=2 x^{3}+6 x^{2}+10 x+135$

$W^{(8)}(G, x)=2 x^{2}+6 x+145 ; \quad W^{(9)}(G, x)=2 x+151$

$\frac{d}{d x} H W(G, x, y) / x=1, y=(1,1,1,1,1 \ldots .)=1661=.R(G)$

\begin{tabular}{|c|c|c|c|c|}
\hline $\mathbf{n}$ & Diagram & WV & $H W V$ & W/R \\
\hline 1 & & $(6,12,9)$ & $(27,12,3)$ & $27 / 42$ \\
\hline 2 & & $\begin{array}{l}(11,28,36, \\
24,10)\end{array}$ & $\begin{array}{l}(109,64,30, \\
10,2)\end{array}$ & $109 / 215$ \\
\hline
\end{tabular}


P.Gayathri and T.Ragavan

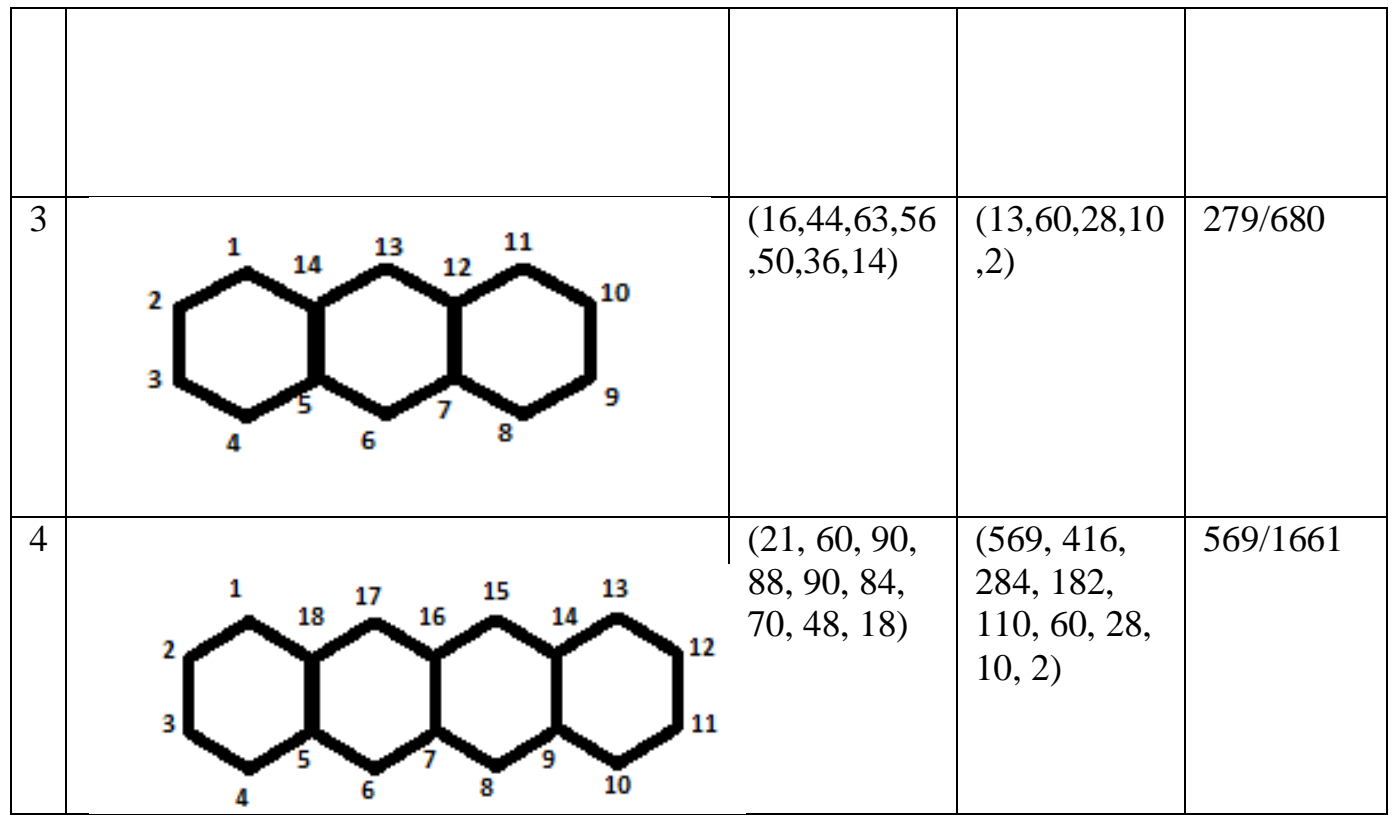

Table 1

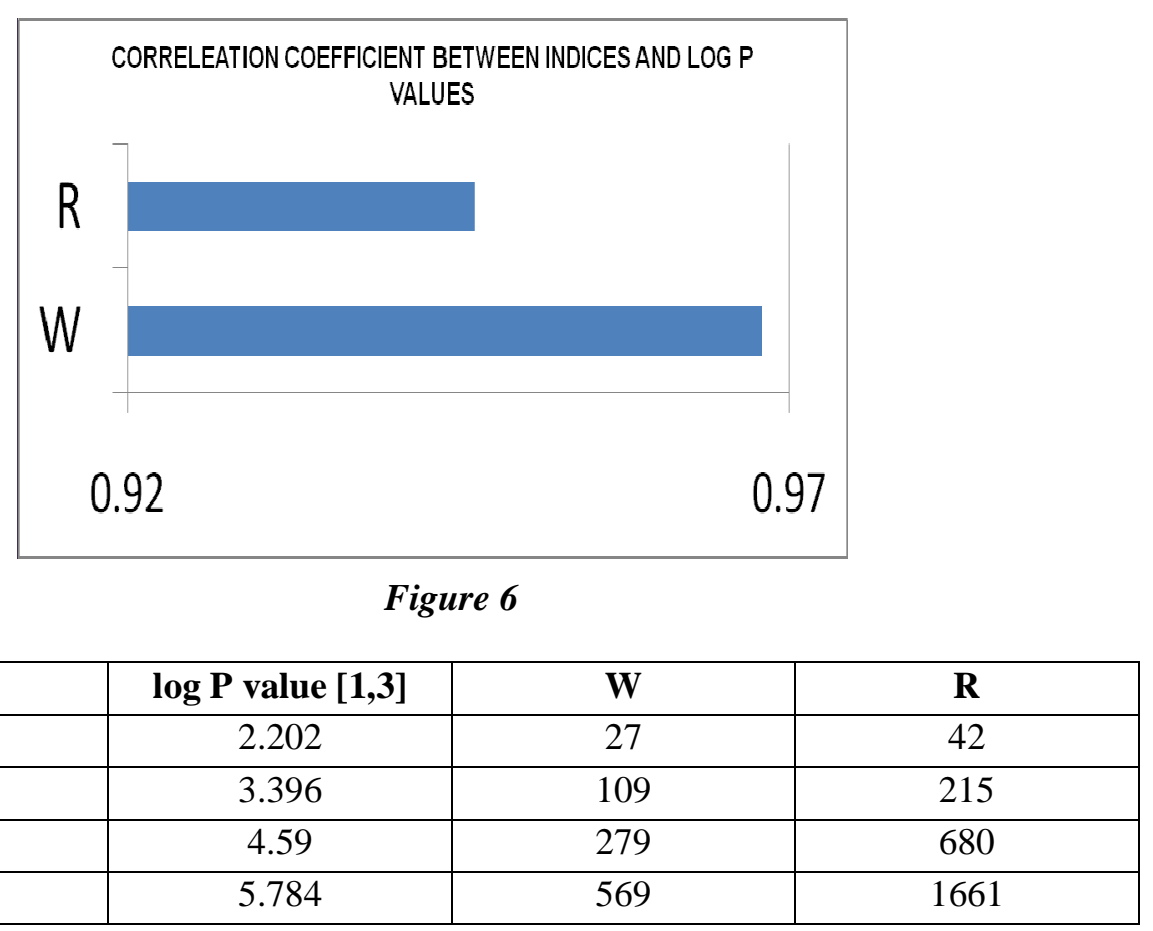

Table 2

\section{Conclusion}

In the research paper, the correlation analysis is given between the results of topological indices obtained and its $\log \mathrm{p}$ value. The topological indices namely Wiener vector, 


\section{Wiener Vector, Hyper-Wiener Vector, Wiener Number and Hyper-Wiener Number of Molecular Graphs}

Hyper-Wiener Vector, Wiener Matrix Sequence, Hyper Wiener Matrix Sequence, Wiener Polynomial Sequence and Hyper-Wiener Polynomial, Wiener number and Hyper Wiener number of Benzenoid hydrocarbons molecular graphs are evaluated and given in neat and easily executable format. The correlation coefficient shows that the present findings are having the highest accuracy to predict physical properties of the chemical. These results will be helpful the study of structure-property relationship for the chemists.

\section{REFERENCES}

1. Padmakar V. Khadikar, et al. Prediction of lipophilicity of polyacenes using quantitative structure-activity relationships, Bioorganic and Medical Chemistry, 10 (2002) 3499-3507.

2. D. Andrijevic, On b-open sets, Mat. Vesnik, 48(1996) 59-64.

3. P.Gayathri and T.Ragavan, Wiener matrix sequence, Hyper-Wiener Vector, Wiener polynomial sequence and Hyper-Wiener polynomial of Bi-phenylene, International Journal of Innovative Research in Science,Engineeringand Technology, 6 (2017) 16998-17005

4. Immadesetty Pothu Raju, Highly correlated Wiener polarity Index: A model to predict $\log$ P, International Journal of Advances in Applied Sciences, 3(2014) 19-24.

5. A.Behmaram and H.Yousefi-Azari, Further results on Wiener polarity index of graphs, Iranian Journal of Mathematical Chemistry, 2 (2011) 67-70.

6. H.Wiener, Structural determination of paraffin boiling points, J Am Chemsoc, 69(1) (1947) 17-20.

7. P.Gayathri, U.Priyanka, S.Sandhiya, S.Sunandha, K.R.Subramanian, M-Polynomials of Penta-Chains, Journal of Ultra Scientist of Physical Sciences, 29(4) (2017) 164168.

8. P.Gayathri, K.R.Subramanian, The PI (Padmakar-Ivan) Index of Polyominoes, International Journal of Discrete Mathematics, 1(1) (2016) 1-4.

9. Bolian Liu, HuoquanHou, Yufei Huang, On the wiener polarity index of trees with maximum degree or given number of leaves, Computers and Mathematics with applications, 60 (2010) 2053-2057.

10. P.Gayathri, U.Priyanka, S.Sandhiya, A significant computation for finding PI index of Phenylene, Journal of Ultra Chemistry, 13(3) (2017) 60-64.

11. P.Gayathri, U.Priyanka, Degree based topological indices of banana tree graph, International Journal of Current Research and Modern Education, (2017) 13-24.

12. Ante.Milicevic, Sonja n. On variable Zagreb indices, Croatica Chemica Acta, 77(12) (2004) 97-101.

13. P.Gayathri, U.Priyanka, Degree based topological indices of linear Phenylene, International Journal of Innovative Research in Science Engineering and Technology, 6(8) (2017).

14. S.Wang, Bolian Liu, A method of calculating the Edge-Szeged index if Hexagonal Chain, Match Commun Math Comput Chem, 68 (2012) 91-96.

15. T.Doslic, On Discriminative of Zagreb indices, Iranian Journal of Mathematical Chemistry, 3 (2012) 25-34. 